\title{
A JPEG backward-compatible HDR image compression
}

\author{
Pavel Korshunov ${ }^{a}$, and Touradj Ebrahimi ${ }^{a}$ \\ ${ }^{a}$ Multimedia Signal Processing Group, EPFL, Switzerland;
}

\begin{abstract}
High Dynamic Range (HDR) imaging is expected to become one of the technologies that could shape next generation of consumer digital photography. Manufacturers are rolling out cameras and displays capable of capturing and rendering HDR images. The popularity and full public adoption of HDR content is however hindered by the lack of standards in evaluation of quality, file formats, and compression, as well as large legacy base of Low Dynamic Range (LDR) displays that are unable to render HDR. To facilitate wide spread of HDR usage, the backward compatibility of HDR technology with commonly used legacy image storage, rendering, and compression is necessary. Although many tone-mapping algorithms were developed for generating viewable LDR images from HDR content, there is no consensus on which algorithm to use and under which conditions. This paper, via a series of subjective evaluations, demonstrates the dependency of perceived quality of the tonemapped LDR images on environmental parameters and image content. Based on the results of subjective tests, it proposes to extend JPEG file format, as the most popular image format, in a backward compatible manner to also deal with HDR pictures. To this end, the paper provides an architecture to achieve such backward compatibility with JPEG and demonstrates efficiency of a simple implementation of this framework when compared to the state of the art HDR image compression.
\end{abstract}

Keywords: High dynamic range, HDR, backward compatibility, JPEG, subjective tests, tone-mapping

\section{INTRODUCTION}

High Dynamic Range (HDR) imaging is an increasingly popular topic and has been the focus of attention in both scientific and artistic communities for several years. Several proprietary image and video compression algorithms have been proposed in the literature for compression of HDR content. Among the most popular are those that are backward compatible with existing Low Dynamic Range (LDR) image and video compression standards. ${ }^{1,2}$ More recently, some standardization committees have also rolled out compression algorithms that can handle both HDR and LDR content, such as JPEG 2000 and JPEG XR for images, and MPEG-4 and H.264/AVC for video, providing limited support of high dynamic range. However, despite wide developments there is a lack of standards in the evaluation of quality and compression of HDR images and video. Most such solutions are ad hoc patches to existing algorithms, where care has been mainly devoted to extend existing approaches to compression and storage adapting them to cope with HDR content, without an explicit exploitation of knowledge of the human visual system, statistical properties of HDR content, or application-specific parameters. Such approach leads to sub-optimal compression solutions for HDR imaging.

Many different subjective evaluations have been previously performed to compare different tone-mapping operators for HDR images and video. Main focus of these studies was either on determining a more superior approach to tone-mapping or establishing an evaluation methodology for subjective evaluation of HDR content. As different evaluations result in different sets of best tone-mapping algorithms, it demonstrates that other factors may also affect perceptual quality of the resulted LDR images.

This paper will start by analyzing the impact of contextual and environmental parameters on perception of quality in HDR image and video, such as display type, size, contrast, and brightness characteristics, as well as the type of content, in different surrounding lighting conditions. We have designed a comprehensive methodology for subjective evaluation of quality and conducted supporting set of subjective tests to build a model of the perception of quality of HDR content by human subjects in various contexts and environments. The test-bed and infrastructure used in the paper consist of displays with different sizes and characteristics, such as mobile

emails: pavel.korshunov@epfl.ch, touradj.ebrahimi@epfl.ch 
phones, tablets, and large monitors. Environmental conditions and contextual information include the amount of environmental lighting, the way subjects view the images, and the backlit light of displays, as well as their size and contrast.

One main novelty of this approach is evident when considering that the majority of current evaluation work in HDR ignores the context and environmental factors. By varying different environmental parameters, one could see how these factors affect the perceptual quality of the content.

The results of the subjective evaluation motivated us to develop an HDR compression algorithm that takes into account the statistical properties of the environment, the content, and used tone-mapping algorithm. We consider JPEG format for backward compatibility as it is the most popular format for images. We propose a generic compression scheme accommodating the above mentioned contextual and environmental parameters into the encoding and decoding design. We then implement a simple version of the compression scheme, which results in a more efficient encoding in terms of size of compressed data, demonstrating the advantage of utilizing the knowledge about the used tone-mapping algorithm.

\section{RELATED WORK}

Several subjective evaluation studies have been conducted in literature to compare different tone-mapping methods for HDR images. One of the first subjective evaluations of HDR images was performed by Ledda et al. ${ }^{3}$ The authors used paired comparison to evaluate the perceptual quality of six different tone-mapping algorithms. An HDR display was used as reference display for 48 subjects. The focus of this work was on the evaluation methodology for the subjective comparison of HDR images in a controlled environment. The evaluations provided the performance ranking of different tone-mapping algorithms leading to different perceptual qualities in color and gray images.

Yoshida et al. ${ }^{4}$ evaluated seven different tone-mapping algorithms via subjective tests to rate the resulted images with regards to their naturalness, contrast, brightness and details of the reproduction in bright and dark regions. A prior study was conducted to find out the best tuning parameters for each tone-mapping. The overall goal was to see if different tone-mapped images are perceived differently. The results show that brightness was the largest differentiator and local-based tone-mapping is better for details in brighter regions. Three methods were concluded as producing the most natural LDR from HDR images.

The focus in the work by Park and Montag ${ }^{5}$ was on the scientific images (astronomic, medical, infrared, radar), where 9 tone-mapping algorithms were evaluated. Subjective tests with 25 subjects were conducted in a typical controlled environment, where only default parameters were used. Paired comparison was used to (i) find which tone-mapping is preferable perceptually, and (ii) to determined which operator is more "scientifically useful". Results concluded that there were no correlations between perceptual preference and scientific usefulness. One method was selected as performing best for both criteria.

Kuang et al. ${ }^{6}$ studied the overall preference of tone-mapping algorithms (9 in total) and their accuracy when compared to actual world scenes, from which they were captured. The focus was on the evaluation methodology. Three subjective studies (paired comparison, rating-scale method, and real-world scenes method) were performed with fixed environmental parameters (lighting, luminance, screen sizes, etc.) with 19 to 23 subjects. In rating-scale method, the following features were investigated: details, shadow detail, overall contrast, sharpness, colorfulness, and lack of artifacts. Different evaluation methods were compared with each other. Bilateral filter was shown to outperform other tone-mapping algorithms, which seems to indicate a different conclusion when compared to other papers mentioned above.

Cadík et $a .^{7}$ studied how tone-mapping algorithms (14 in total) affect the comprehensive set of image attributes, namely and mainly, brightness, contrast, color reproduction, reproduction of details, and artifacts. They proposed an overall quality metric as a combination of the image attributes. Two subjective tests with 20 subjects in total were performed: (i) with real world reference, and (ii) without a reference. Authors did not find any significant differences between the two testing methodologies in terms of results. Global tone-mapping algorithm was shown to be superior.

Annighöfer et $a l .{ }^{8}$ evaluated 8 tone-mapping operators against linear tone-mapping with 51 subjects. The focus of their work was on objective metrics that match subjective results. In addition, HDR images with 
restricted bit resolution were used (with different base exposures). Three tone-mapping algorithms were found performing well, consistent with previous studies. However, performance of each operator was found to be content dependent. Naka-Rushton RMS objective metric demonstrated to be the most robust.

The study by Mai et al. ${ }^{9}$ extended the subjective evaluations to 3D HDR content on 3D stereoscopic displays with active shutters. The main objective of this work was to study which image attributes contribute to a good 3D representation and what are the differences with $2 \mathrm{D}$ case. A pilot study was conducted to find best possible parameters for the tone-mapping algorithms. 3D effect and overall quality of the tone-mapped images were evaluated, including contrast, naturalness, sharpness, and detail reproduction image attributes. Paired comparison and rating scale were used. Global tone-mapping operators were found to outperform others (same as in 2D case). In addition a high correlation between brightness and 3D effect was found in the results.

Compared to all these subjective tests, the goal of our study is not to find the best tone-mapping algorithm but to demonstrate the importance of other factors, such as display size, type of content, and environment on the quality of resulted tone-mapped image, etc. And the findings of these tests can guide the development of more efficient compression algorithms for HDR images and better file formats. Although, there are many file formats for HDR images, as with tone-mapping algorithms, none stands out consistently, when compared to others. And there is a lack of widely used standard for both format and compression of HDR images.

One popular format is Radiance, first proposed by Greg Ward in Ref. 10. This format encodes floating point HDR pixels represented in 32 bits RGBE format, with red, green, and blue mantissas, plus a common exponent each stored as 8 bits integers. This format was supported by released Radiance software, ${ }^{11}$ which is a comprehensive set of tools for image manipulation and rendering, implemented under public license, significantly boosting the popularity of the format. This format uses simple run length encoding to losslessly compress an image.

The same Greg Ward also proposed a JPEG backward compression algorithm and file format called JPEGHDR,${ }^{1}$ which is one of the few lossy HDR compression schemes and is the main JPEG backward compatible scheme widely used. JPEG-HDR compresses an image into a JPEG tone-mapped version of that HDR image (any tone-mapping operator is allowed to be used) and a residual data stored as an extension of JPEG. This approach allows any conventional JPEG decoder to render a tone-mapped version, and for JPEG-HDR aware software to reconstruct the original HDR image. In this paper, we agree with the idea of having JPEG backward compatible HDR compression and format, but propose having a more general view on the compression algorithm that would allow to have both lossless and lossy compression, as well as to achieve a more efficient compression and flexibility, given how the research on compression has advanced since JPEG-HDR was first introduced.

JPEG XR and JPEG 2000 are two standards that are capable of storing and compressing HDR content. The main problem with these formats, however, is that they are not very popular and have little software support for HDR content. We believe that, since JPEG is currently a de facto and most popular imaging format, any next generation format and compression for HDR content is bound to offer backward compatibility with JPEG in order to increase chances of use by public.

\section{SUBJECTIVE EVALUATIONS}

The goal of this section is to analyze the suitability of the most common image and video quality evaluation methods for the subjective evaluation of HDR content and to adapt/extend these methods to take into the account contextual and environmental information. We study the effect of the environmental conditions, display characteristics, and content types on the perceptual quality of HDR images. We have designed a comprehensive methodology for subjective evaluation of quality and conducted supporting set of subjective tests to build a model of the perception of quality of HDR content by human subjects in various contexts and environments.

\subsection{Test material and tone-mapping algorithms}

As the aim of the subjective study was not to find the best tone-mapping algorithm but to understand how they perform in different conditions, we selected the following five tone-mapping operators: "drago" by Drago et al. ${ }^{12}$ "mantiuk" by Mantiuk et al. ${ }^{13}$ "reinhard" by Reinhard and Devlin, ${ }^{14}$ "icam" by Fairchild and Johnson, ${ }^{15}$ and "log" as a simple logarithm-based operator. The implementation provided in library "pfstools" was used 
for the first three operators and code provided in the book by Reinhard et al. ${ }^{16}$ was used for the last two. These algorithms were selected to have representation of different approaches to tone-mapping, such as global operators (logarithm-based and "drago"), based on local information ("reinhard" and "icam"), and operators utilizing properties of human visual system ("mantiuk").

To test the selected tone-mapping operators, we used four images (see Fig. 1) from the collection provided in the book by Reinhard et al., ${ }^{16}$ as the images typically used for evaluation of tone-mapping operators have very low resolution for today's monitors. The smallest resolute of the chosen images is $1840 \times 1224$. Images are stored in Radiance file format ${ }^{10}$ with 32 bits per pixel. Therefore, tone-mapping operators map floating point values of HDR content in RGBE representation into 24 bits RGB. The images were also selected in such a way to have different representation in terms of content type (indoor or outdoor) and luminance range (night and day shots).

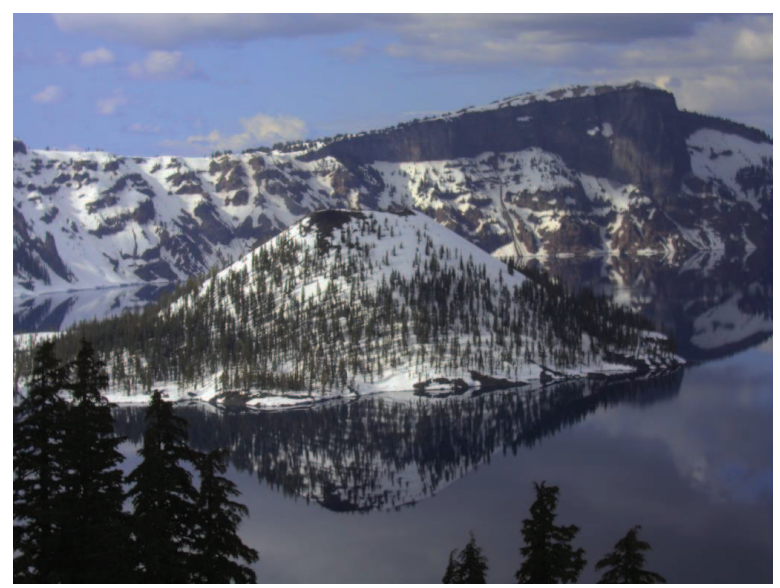

(a)

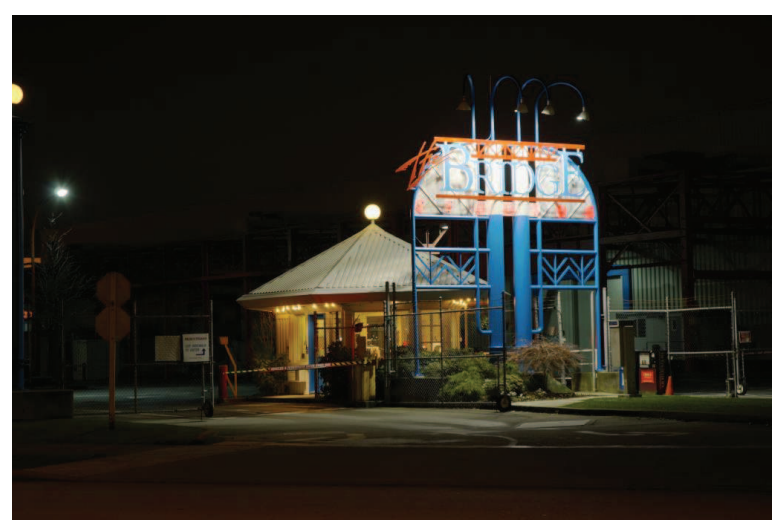

(c)

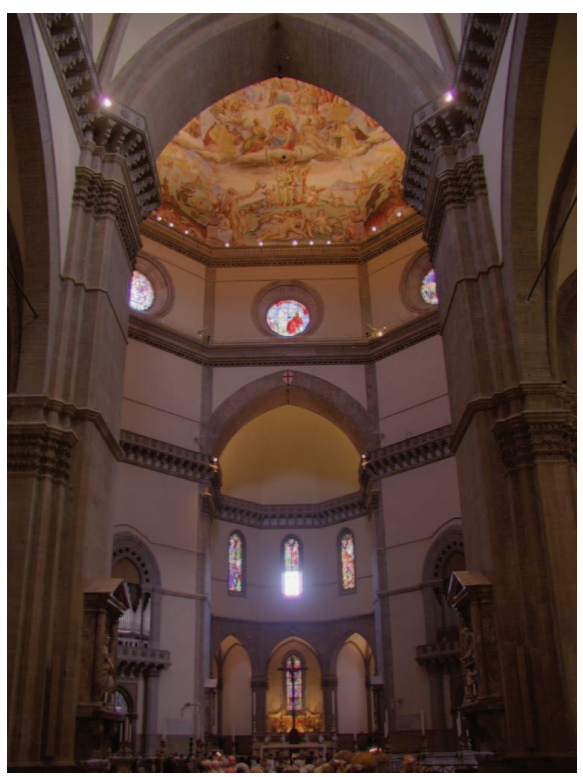

(b)

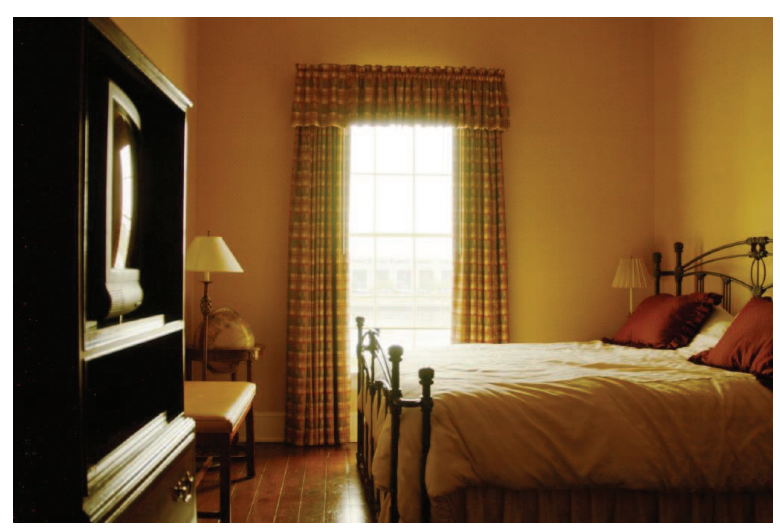

(d)

Figure 1: Four HDR images: "CraterLake1"(a), "Duomo1"(b), "BridgeStudios2"(c), and "Room"(d) were used in the experiments.

\subsection{Test environment and methodology}

We have conducted subjective tests as pairwise comparison of different tone-mapping operators. For pairwise comparison of five operators with four test images, 40 pairs in total is necessary. 
The test-bed and infrastructure used in the paper consist of displays with different sizes and characteristics, such as mobile phones, tablets, and large monitors. Environmental conditions and contextual information include the amount of the backlit light of displays, as well as their size and contrast. In practice, the environmental ambient light can be captured by either frontal camera of the device (in case of mobile phone and tablet) or a camera (in case of monitor).

The tests were performed in a laboratory for professional subjective tests when using a monitor and in a typical office environment when tablets and mobile phones were used. The test room was equipped with a 30" LCD Eizo monitor with resolution $2560 \times 1600$. The ambient lighting was obtained with neon lamps of $6500 \mathrm{~K}$ color temperature and the walls color were painted mid gray 128, as recommended in Ref. 17 . The luminance of the Eizo screen was set with EyeOne Display 2 calibration tool to $120 \mathrm{~cd} / \mathrm{m}^{2}$. We used first generation of iPad with resolution $1024 \times 768$ in experiments with tablets, and Samsung Galaxy S with resolution $800 \times 480$ for tests with mobile phones. The office for testing with tablets and mobile phones had a typical lighting of about 500 lux, and the brightness was turned to maximum in both types of devices.

With the monitor tests were performed in a passive mode as follows. Each subject was sitting in front of the monitor at a distance 2 to 3 times the height of the stimuli. A pair of test stimuli in the same comparison set were played one-after-another. All possible pairs in each comparison set were used for comparison in order to obtain complete winning frequency matrices. Since the monitor used had a native resolution of $2560 \times 1600$, images could fit in the horizontal space of the display. Each subject was asked to choose which stimulus had better quality between the two presented stimuli and to mark the answer between first, same, and second on the score sheet. Each stimuli of the pair was displayed for 7 seconds and 5 seconds was given to vote after the pair. Each subject had two separate test sessions, each of which contained 20 pairs of stimuli. A training session took place before the test sessions. Twenty subjects (12 males and 8 females) having normal or corrected-to-normal vision participated in the tests with ages ranging from 20 years old to 61 years old and a median age of 25 .

The experiments with mobile phones and tablets were performed in similar ways, only users were allowed to scroll through the pairs of images by themselves, enabling a more realistic active mode in subjective evaluations in such contexts.

The test images (see Fig. 1) in original Radiance format were resized to fit different resolutions of monitors, tablets, and mobile phones. Then, the tone-mapping operators were run on each image with default settings to produce LDR versions in JPEG format, which were used in the pairwise comparisons.

\subsection{Evaluation Results}

To have a better understanding of the subjective tests results, we first computed the number of subjective votes for each compared pair of tone-mapping operators, as presented in Fig. 2-5. The pairs of tone-mapping algorithms are displayed on vertical axis, with "drago" denoted as "dr", "mantiuk" as "ma", "reinhard" as "ra", "icam" as "ic", and logarithmic as "lg". Values representing horizontal bars are computed as follows (in the order from left to right): the number of subjects that favored first algorithm of the compared pair, the number of subjects voting that algorithms were the same, and the number of subjects that favored the second algorithm. All these values are divided by the total number of subjects that participated in tests for each device, resulting in probability value. Each figure presents results for each image with three subfigures corresponding to three devices (in order of appearance): Eizo monitor, iPad tablet, and Samsung mobile phone, on which the evaluations were performed.

As can be noted from these figures, there is a variety of scores across both devices and different images, with variety being more significant across images. It means that content of the images (the luminance range, whether it is a day or night shot, variety of details, etc.) plays more significant role in determining which tone-mapping suits better.

To clearly illustrate which tone-mapping outperforms in which conditions, we have computed judging probabilities from the subjective tests using BTL (Bradley-Terry-Luce) model ${ }^{18}$ which is a commonly applied model for comparison of pairwise data. The judging probabilities per tone-mapping operator are presented in Fig. 6-9. From these figures, one can identify rather easily the most and the least favorite tone-mapping algorithms for each device and for each image. From the selected set of tone-mapping operators, the one by Drago et al. performs the best in most scenarios, while in some cases, such as for "Duomo1" image (central and left graphs 

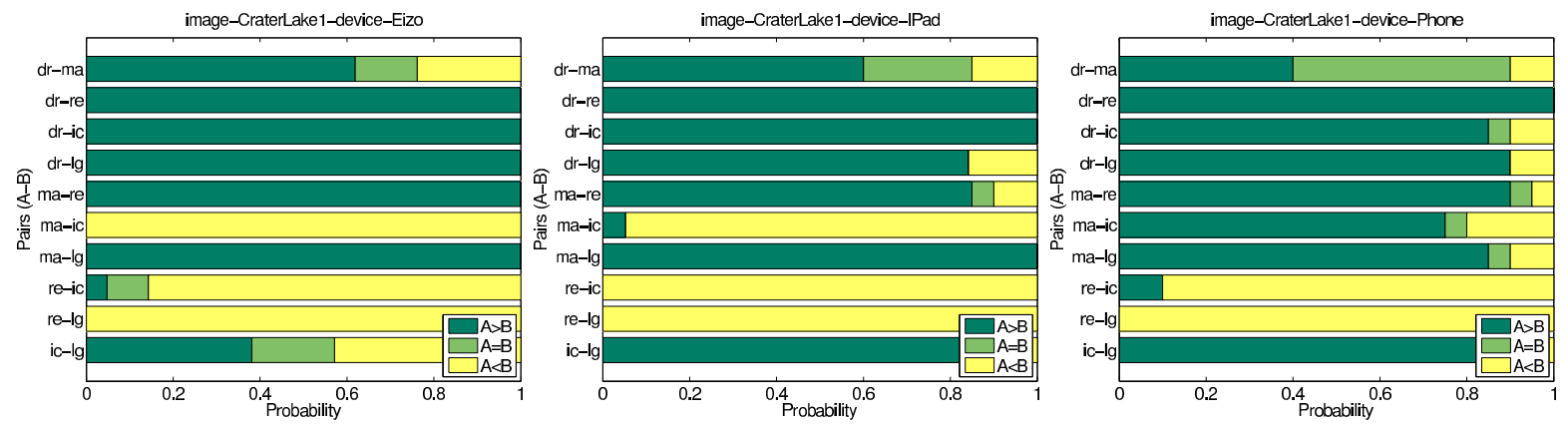

Figure 2: Scores for each compared pair of tone-mapping operators for "CraterLake1" image.
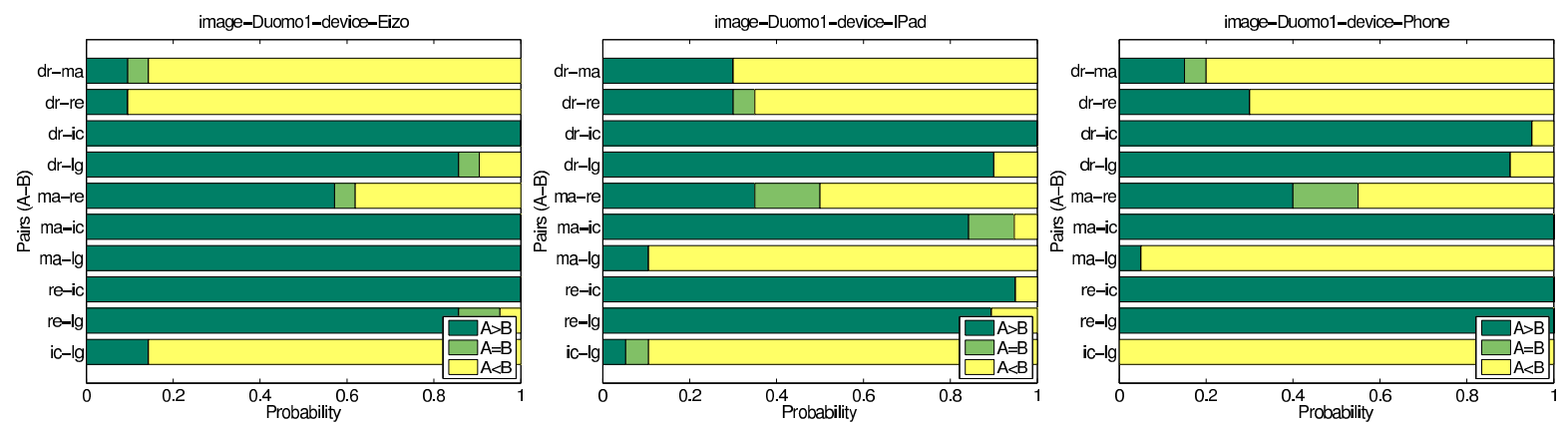

Figure 3: Scores for each compared pair of tone-mapping operators for "Duomo1" image.
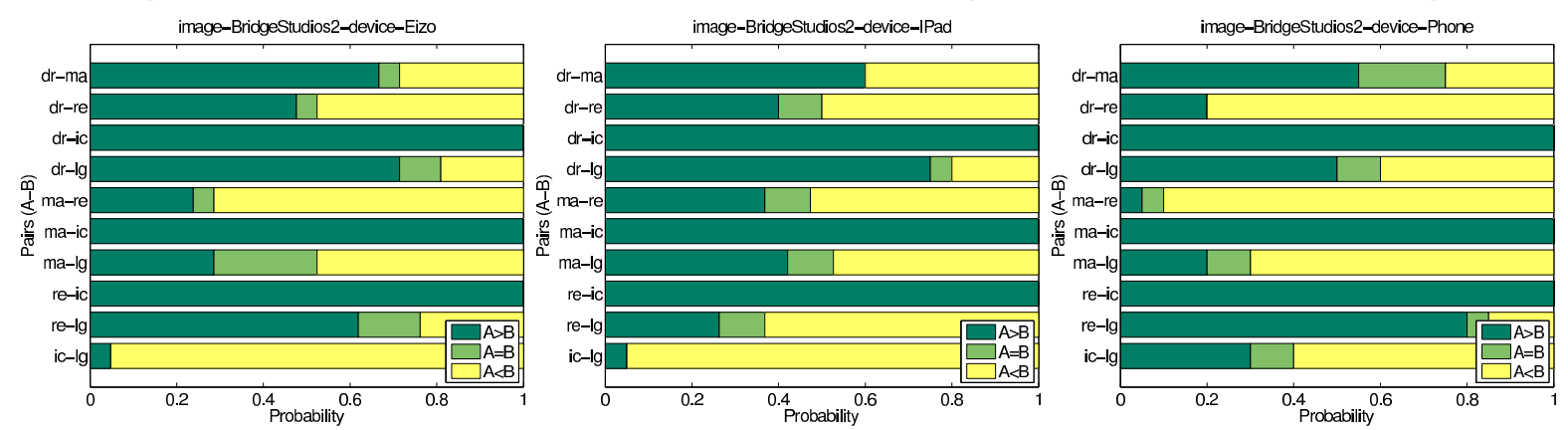

Figure 4: Scores for each compared pair of tone-mapping operators for "BridgeStudios2" image.
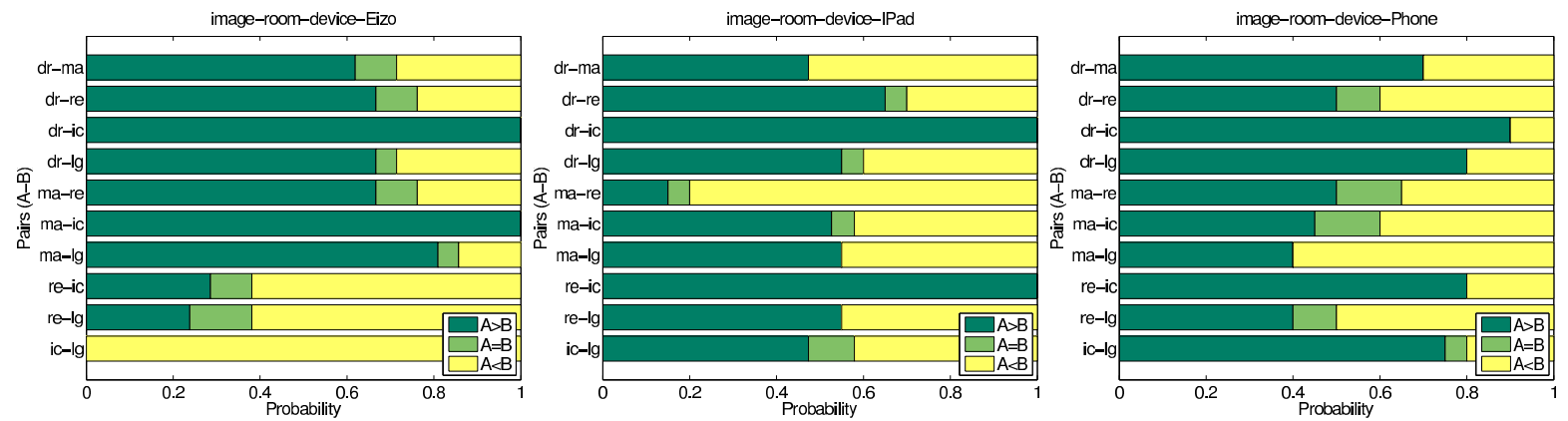

Figure 5: Scores for each compared pair of tone-mapping operators for "room" image.

in Fig. 7 for Eizo monitor and mobile phone) and for "BridgeStudio2" image (left graph in Fig. 8 for mobile phone), it shows poor performance. 

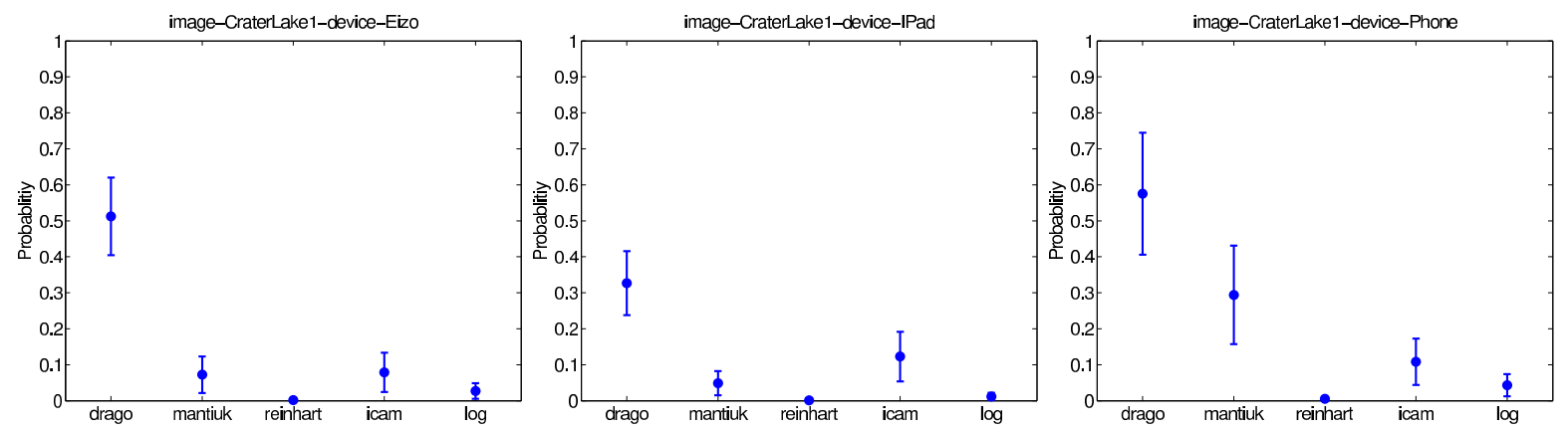

Figure 6: Overall subjective scores of tone-mapping operators for "CraterLake1" image.
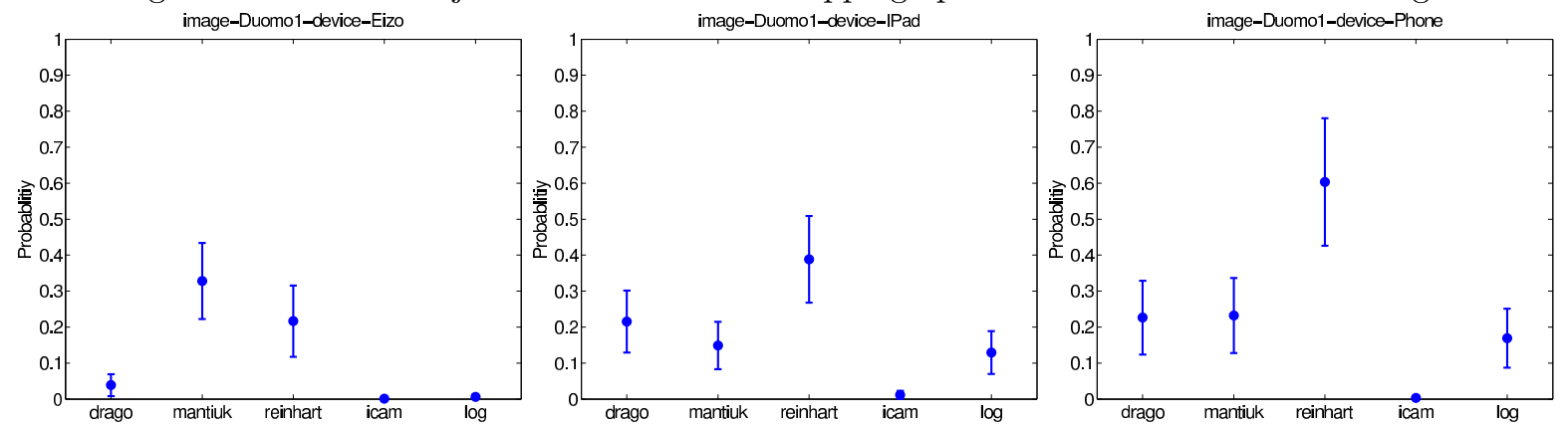

Figure 7: Overall subjective scores of tone-mapping operators for "Duomo1" image.
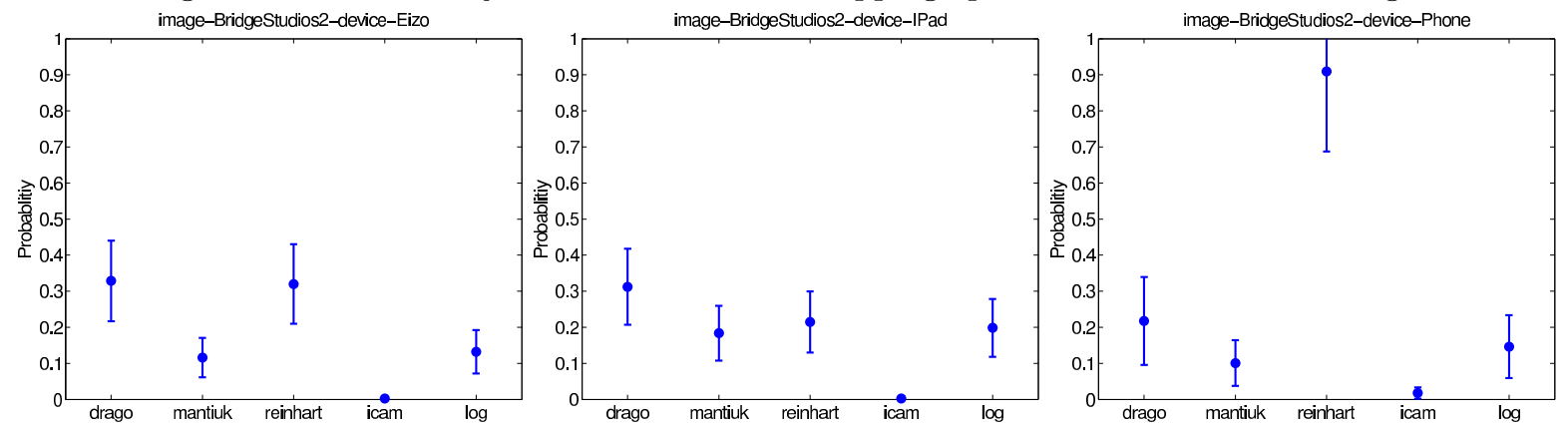

Figure 8: Overall subjective scores of tone-mapping operators for "BridgeStudios2" image.
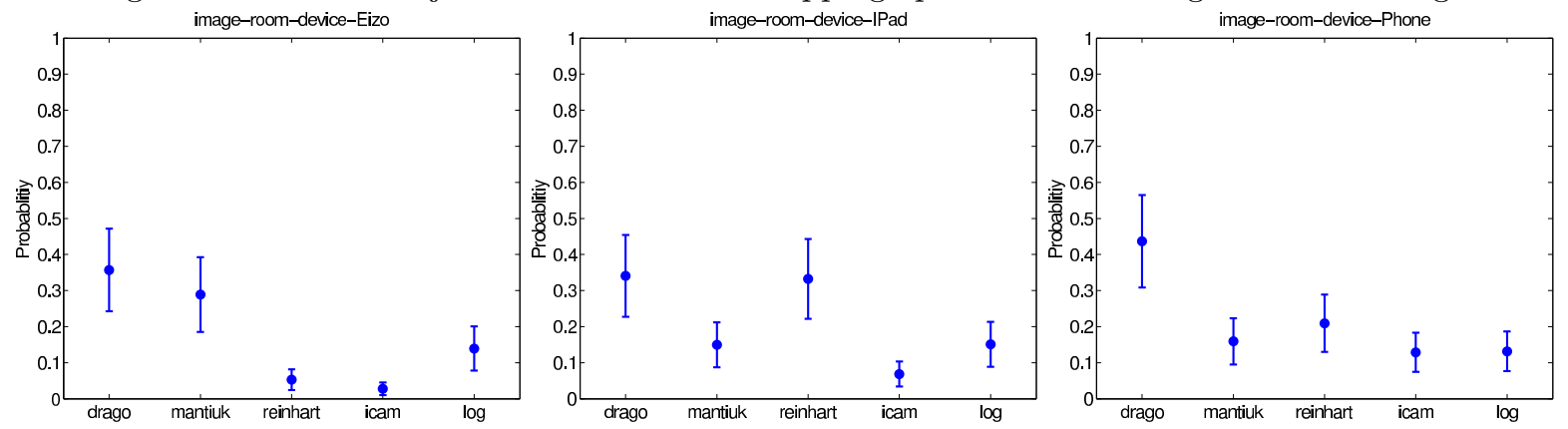

Figure 9: Overall subjective scores of tone-mapping operators for "Room" image.

\section{PROPOSED HDR IMAGE COMPRESSION}

The subjective evaluations results reported in the previous section can guide the design of an efficient JPEG backward compatible compression beyond the state of the art. The subjective scores indicate that to be opti- 
mal, and adaptive to different environments, devices and contents, a mechanism is required for selection of an appropriate tone-mapping operation when converting an HDR image to LDR for display on conventional devices available to consumers. JPEG is selected to represent LDR version of the HDR image, because of the wide-spread use of this format and availability of viewers able to handle images in JPEG. Although a few proprietary JPEG backward compatible HDR compression formats have already been proposed in the state of the art, they often do not explicitly take into account the perceptual quality, nor context- and environmental parameters in their design. Our subjective evaluations described above indicate that such parameters should be considered when selecting a tone-mapping operation to convert an HDR to an LDR image.

\subsection{Architecture of encoder and decoder}

Fig. 10 describes the generic block diagram of the encoder architecture proposed in this paper for HDR image compression, with the feature of being JPEG backward compatible, while offering a more optimal solution when compared to state of the art. By JPEG backward compatibility, we mean that when the resulting bitstream is fed into a conventional JPEG decoder, the latter can decode it into an image and display the content as an LDR version of the original HDR image.

In this diagram, $T M O_{c 1}$ refers to an appropriate tone-mapping operation which converts the input HDR image into a format suitable for JPEG compression and decompression. In particular, the most widely used JPEG compression format relies on a YUV color image representation, with all three components coded with 8 unsigned integer bits, and where $\mathrm{U}$ and $\mathrm{V}$ components are sub-sampled by a factor of 2 in both horizontal and vertical directions, when compared to $\mathrm{Y}$ component. The selection of $T M O_{c 1}$ is made by the encoder based on any consideration, and for the rest of this paper we simply assume that it can be any tone-mapping algorithm. In this sense, the proposed approach is designed to cope with any tone-mapping algorithm, as it is also the case with some existing solutions in the state of the art, such as JPEG-HDR. TMO $O_{2}$ indicates an optional tone-mapping operation which could exist in the encoder, in case the representation of the input HDR image is different from the internal HDR content representation in the codec, or when the application requires a different HDR representation than that of the input image. In particular, the color components and bit depth representations of the input HDR image may be different from that used internally in the codec. This component brings an additional flexibility to the approach proposed and allows to cope with a wide variety of HDR images and situations. JPEG and JPEG ${ }^{-1}$ indicate a conventional JPEG compression and decompression. In this

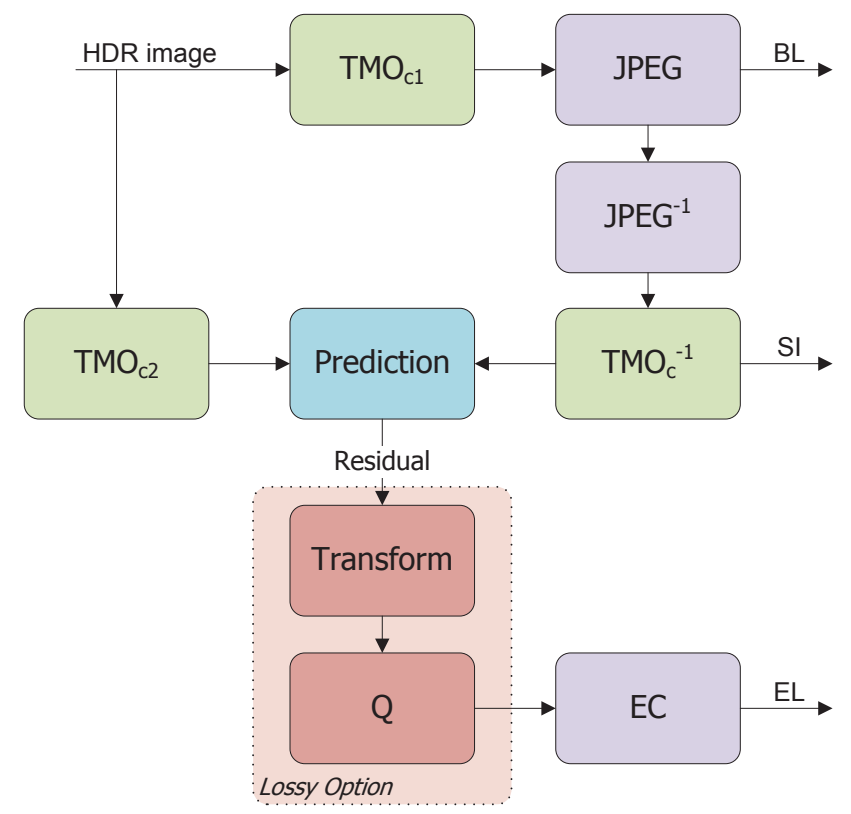

Figure 10: Scheme of JPEG backward compatible encoding process. 


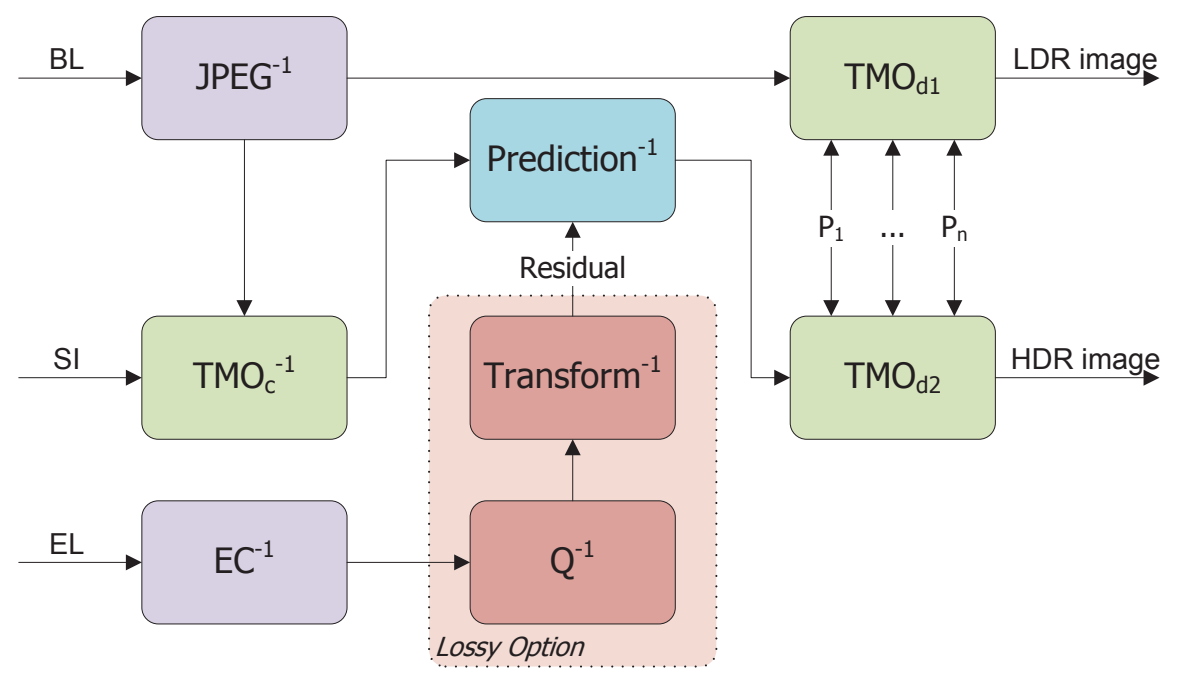

Figure 11: Scheme of JPEG backward compatible decoding process.

context, no further assumption is made and the compression ratio, or any other JPEG compression parameters such as the choice of quality factor, quantization and entropy coding tables, or any pre- and post- processing for the purpose of JPEG compression and decompression are left to the encoder, with the largest degree of flexibility. The $B L$ indicates the baseline portion of the resulting bitstream and consists of a fully compatible JPEG format, readable by any compliant JPEG decoder. As in many extensions of JPEG, the additional bit stream necessary for decoding the HDR image will be included in the baseline JPEG format thanks to an appropriate APPn application marker as proposed in JPEG standard.

One of the essential components of the proposed algorithm is the $T M O_{c}^{-1}$, which refers to an operation, which will convert the JPEG decoded LDR image to an appropriate HDR version. In principle this operation can be anything in a generic situation, but the efficiency of the proposed algorithm largely depends on the choice of this operation. In particular, one could observe that a $T M O_{c}^{-1}$ algorithm that can lead to an as small as possible residual HDR (see Sec. 4.2) will lead to very efficient compression performance. The proposed approach leaves the choice of this operation to the encoder and provides a mechanism to inform the decoder by means of a side information $(S I)$ how the operation should be reproduced in the decoder. One can imagine that efficient $T M O_{c}^{-1}$ operations, depending on the content on one hand and the $T M O_{c 1}$ on the other hand, would lead to more efficient solutions. This is a major difference between the proposed solution in this paper and other state of the art solutions for HDR image compression. The output of $T M O_{c}^{-1}$ is then fed into a prediction component, which will calculate the residual HDR portion of the input image. The prediction can be either in the form of a differential, a ratio, or a more complex mechanism. The residual image can then be compressed either in a lossless fashion using an appropriate entropy coding, if a lossless solution is desired, or transformed, quantized, and then entropy coded, if lossy compression is used. The enhancement layer $(E L)$ containing the residual HDR portion of the input image, along with the $S I$ is then embedded inside the JPEG compressed file format, using APPn marker as indicated earlier.

The proposed decoder is depicted in Fig. 11, and essentially provides the dual operations of the encoder. In addition, and in order to be able to cope with context and environmental parameters (denoted as $p_{1}, \ldots, p_{n}$ ), additional operations denoted as $T M O_{d 1}$ and $T M O_{d 1}$ can be applied as post-processing, taking into account also other encoding parameters aiming as maximizing the visual quality of LDR and/or HDR decoded images.

\subsection{Illustrative implementation examples and results}

To illustrate the compression scheme presented in Fig. 10 and Fig. 11, we implemented two variants of a simple codec based on the proposed solution. The following simple tone-mapping algorithms were used in each variant: (i) gamma correction and (ii) logarithm-based operator. The main goal of this implementation is to demonstrate 
that the knowledge of tone-mapping used to produce the JPEG image from the original HDR can help achieving efficient compression even via simple means.

Although, the proposed solution can cope with both lossless and lossy compression, in our illustration, we focus only on lossy scenario. For implementation of various components, we relied on HDR toolbox* freely available for Matlab. Since we only focus on lossy case, we compare the results against JPEG-HDR lossy algorithm, ${ }^{1}$ an adaptation of the JPEG 2000 for HDR content proposed in Ref. 19, and JPEG XR compression standard implemented in XnView software ${ }^{\dagger}$.

The detailed process of HDR image compression in a JPEG backward compatible manner consists of the following steps:

1. Application of $T M O_{c 1}$ (gamma correction or logarithm-based) on the HDR input image and JPEG compression. For logarithm $T M O_{c 1}$, we will also maintain maximal luminance value, which is a constant value.

2. Application of an inverse $T M O_{c}^{-1}$ (gamma correction or logarithm-based) to the decoded JPEG image in order to obtain an approximation of the original HDR image, denoted by $H D R j$.

3. Conversion of both original HDR image and $H D R j$ to $\mathrm{YCbCr}$ format, illustrating $T M O_{c 2}$ and final processing stage in $T M O_{c}^{-1}$.

4. Formation of residual of the luminance component $\mathrm{Y}$ as the difference between $\mathrm{Y}$ component of the original HDR image and Y component of predicted $H D R j$ obtained from JPEG decoding and $T M O_{c}^{-1}$.

5. Compression of the residual Y component as a grey image in 16 bits integer format using lossless JPEG2000 compression algorithm ${ }^{\ddagger}$.

Table 1: Comparison of lossy compression methods for image "Room".

\begin{tabular}{|l|c|c|c|c|c|}
\hline Compression & $\begin{array}{c}\text { Data size } \\
\text { (bytes) }\end{array}$ & RMSE Y & RMSE Cb & RMSE Cr & PSNR Y \\
\hline Original & $7,438,076$ & - & - & - & - \\
\hline JPEG-HDR $^{1}$ (quality=100) & $2,262,404$ & 0.0127 & 0.01282 & 0.01305 & 37.92 \\
\hline JPEG 2000 ${ }^{19}$ (rate=1, 8 bits) & $2,069,384$ & 0.0635 & 0.23100 & 0.49203 & 23.95 \\
\hline JPEG XR (YUV444, q=100) & $1,570,385$ & 0.12309 & 0.06418 & 0.16253 & 18.20 \\
\hline Proposed method (GammaTMO) & $1,163,436$ & 0.00266 & 0.01032 & 0.03993 & 51.51 \\
\hline Proposed method (LogTMO) & $1,149,630$ & 0.00179 & 0.01146 & 0.02653 & 54.96 \\
\hline
\end{tabular}

The decoding process follows a similar but dual path when compared to encoding. To make it simple, during decoding, the residual for Y component of the HDR image (represented in YCbCr format) is decoded only in an enhanced resolution, while chroma components are obtained directly from the JPEG version of the image.

These HDR image compression implementation variants are then compared to JPEG-HDR, JPEG XR, and an HDR compression based on JPEG 2000, as reported in Table 1. These results were obtained using "Room" (see Fig. 1d), one of the images from our subjective experiments presented in Sec. 3.3. We compressed this image using each evaluated compression algorithm and computed square root of MSE for each component in $\mathrm{YCbCr}$ representation, as well as PSNR for luminance component. From the results, we can note that the proposed method compresses the image to significantly smaller sizes when compared to JPEG-HDR, JPEG XR, and JPEG 2000 based method, which were all used in lossy mode and the best quality settings possible.

\footnotetext{
${ }^{*}$ HDR toolbox can be downloaded here: http://www.mathworks.com/matlabcentral/linkexchange/links/2792-the-hdrtoolbox

${ }^{\dagger}$ http://www.xnview.com/

${ }^{\ddagger}$ We used OpenSource Jasper implementation of JPEG2000: http://www.ece.uvic.ca/ frodo/jasper/
} 


\section{CONCLUSION}

In this paper, we propose a generic HDR compression algorithm backward compatible with JPEG format. The proposed solution relies on an important observation. By means of rigorous subjective assessments of various tone-mapping algorithms applied to typical HDR images and rendered in various controlled and uncontrolled environments and devices, it is shown that there is no universal tone-mapping algorithm that always stands out when compared to others. The choice of the best algorithm depends on the content, but also on the device used, and other environmental parameters such as back lit lighting, display type and size, environment illumination, etc. These parameters are explicitly taken into account in the proposed solution. Illustrative implementations of the proposed solutions using two simple tone-mapping algorithms show that significant compression efficiency can be obtained when compared to state of the art.

The results presented in this paper can be extended in several directions. First and utmost, extension of performance evaluation to include a larger set of typical HDR images and other more sophisticated tone-mapping algorithms. Second, exploring other HDR image prediction strategies from JPEG decoded LDR image, as well as alternatives compression of residual images both in lossy and lossless fashions. Finally, a rigorous subjective evaluation of both LDR and HDR decoded images obtained and comparisons to state of the art from subjective quality point of view rather than MSE or PSNR as reported in this paper.

\section{ACKNOWLEDGEMENTS}

This work was conducted in the framework of the EC funded Network of Excellence VideoSense, with partial support from COST Action IC1005 - HDRi, and COST Action IC1003 - Qualinet. Help from Shuting Cai in generating some of the HDR and tone-mapped images used in subjective evaluations performed in this paper is also acknowledged.

\section{REFERENCES}

[1] Ward, G. and Simmons, M., "JPEG-HDR: a backwards-compatible, high dynamic range extension to jpeg," ACM SIGGRAPH (2006).

[2] Mantiuk, R., Efremov, A., Myszkowski, K., and Seidel, H.-P., "Backward compatible high dynamic range mpeg video compression," 25, 713-723, SIGGRAPH '06 (2006).

[3] Ledda, P., Chalmers, A., Troscianko, T., and Seetzen, H., "Evaluation of tone mapping operators using a high dynamic range display," ACM SIGGRAPH (2005).

[4] Yoshida, A., Blanz, V., Myszkowski, K., and Seidel, H.-P., "Perceptual evaluation of tone mapping operators with real-world scenes," 5666, 192-203, Human Vision and Electronic Imaging X, IS\&T/SPIE's 17th Annual Symposium on Electronic Imaging (2005).

[5] Park, S. H. and Montag, E. D., "Evaluating tone mapping algorithms for rendering non-pictorial (scientific) high-dynamic-range images," J. Vis. Comun. Image Represent. 18(5), 415-428 (2007).

[6] Kuang, J., Yamaguchi, H., Liu, C., Johnson, G. M., and Fairchild, M. D., "Evaluating hdr rendering algorithms," ACM Trans. Appl. Percept. 4(2) (2007).

[7] Čadík, M., Wimmer, M., Neumann, L., and Artusi, A., "Evaluation of hdr tone mapping methods using essential perceptual attributes," Comput. Graph. 32(3), 330-349 (2008).

[8] Annighöfer, B., Tajbakhsh, T., and Grigat, R.-R., "Prediction of results from subjective evaluation of realtime-capable tone-mapping operators applied to limited high-dynamic-range images," J. Electron. Imaging 19 (2010).

[9] Mai, Z., Doutre, C., Nasiopoulos, P., and Ward, R. K., "Subjective evaluation of tone-mapping methods on 3d images," 1-6, Int. Conf. on Digital Signal Processing (2011).

[10] Ward, G., [Graphics Gems II], ch. Real Pixels, 80-83, Academic Press (1991).

[11] Larson, G. W. and Shakespeare, R., [Rendering With Radiance: The Art And Science Of Lighting Visualization], Booksurge Llc (2004).

[12] Drago, F., Myszkowski, K., Annen, T., and Chiba, N., "Adaptive logarithmic mapping for displaying high contrast scenes," in [Proc. of EUROGRAPHICS 2003], Brunet, P. and Fellner, D. W., eds., Computer Graphics Forum 22(3), 419-426, Blackwell, Granada, Spain (2003). 
[13] Mantiuk, R., Daly, S., and Kerofsky, L., "Display adaptive tone mapping," ACM Trans. Graph. 27, 68:168:10 (Aug. 2008).

[14] Reinhard, E. and Devlin, K., "Dynamic range reduction inspired by photoreceptor physiology," IEEE Transactions on Visualization and Computer Graphics 11, 13-24 (Jan. 2005).

[15] Fairchild, M. D. and Johnson, G. M., "The icam framework for image appearance, image differences, and image quality," Journal of Electronic Imaging 13, 126-138 (2004).

[16] Reinhard, E., Ward, G., Pattanaik, S., Debevec, P., Heidrich, W., and Myszkowski, K., [High Dynamic Range Imaging: Acquisition, Display, and Image-based Lighting], The Morgan Kaufmann series in Computer Graphics, Elsevier (Morgan Kaufmann), Burlington, MA, 2nd ed. (2010).

[17] [Methodology for the Subjective Assessment of the Quality of Television Pictures], Recommendation ITU-R BT.500-11., Geneva, Switzerland (2012).

[18] Bradley, R. A. and Terry, M. E., "Rank analysis of incomplete block designs I: The method of paired comparisons," Biometrika 39, 324-45 (1952).

[19] Xu, R., Pattanaik, S. N., and Hughes, C. E., "High-dynamic-range still-image encoding in jpeg 2000," IEEE Comput. Graph. Appl. 25, 57-64 (Nov. 2005). 